\title{
The Prevalence of Overweight and Obesity Among Women in Jordan: A Risk Factor for Developing Chronic Diseases
}

\author{
Mona Bustami (iD) \\ Khalid Z Matalka ${ }^{2}$ \\ Eyad Mallah (1D ${ }^{3}$ \\ Luay Abu-Qatouseh ${ }^{1}$ \\ Wael Abu Dayyih ${ }^{3}$ \\ Nour Hussein ${ }^{4}$ \\ Nayef Abu Safieh ${ }^{4}$ \\ Yousef Elyyan $\mathbb{1}^{5}$ \\ Nagham Hussein ${ }^{6}$ \\ Tawfiq Arafat $^{7}$ \\ 'Department of Pharmacology and \\ Biomedical Sciences, University of Petra, \\ Amman, II 1 96, Jordan; ${ }^{2}$ Faculty of Health \\ Sciences, American University of Madaba, \\ Madaba, Jordan; ${ }^{3}$ Department of \\ Pharmaceutical Medicinal Chemistry and \\ Pharmacognosy, Faculty of Pharmacy \& \\ Medical Sciences, University of Petra, \\ Amman, II 196, Jordan; ${ }^{4}$ School of \\ Medicine, University of Jordan, Amman, \\ Jordan; ${ }^{5}$ Obstetrics and Gynecology, \\ Jordan University Hospital, Amman, \\ Jordan; ' Istishari Hospital, Amman, \\ Jordan; ${ }^{7}$ Jordan Center for Pharmaceutical \\ Research, Amman, Jordan
}

Objective: The study aimed to investigate the prevalence of obesity among Jordanian women and its association with a wide range of chronic diseases.

Methods: Subjects were enrolled in the present cross-sectional study based on a random drop-off technique at the Obstetrics and Gynecology clinics at Jordan University Hospital. Initially, any female 18 years of age and older was asked to enroll in the study. Relevant data were gathered using a questionnaire composed of 30 questions, and body mass index (BMI) was determined from each participant's weight and height. The following variables were collected: socio-demographic, chronic diseases, and health status. Each variable's frequencies were reported, and the $95 \%$ confidence interval $(95 \% \mathrm{CI})$ for each variable was calculated. For association analysis, Chi-square analysis was performed with an odds ratio (OR) and 95\% CI. Multinomial logistic regression analysis was applied to a combination of independent variables and a dependent condition with covariate factors.

Results: The age-standardized prevalence of overweight/obese Jordanian women was $70.6 \%$ (95\% CI 66.0-74.8\%). On the other hand, the age-standardized prevalence of only obese women was $36.4(95 \% \mathrm{Cl} 31.9-41.2 \%)$. Furthermore, the association between age and overweight/obesity was significant $(\mathrm{p}<0.0001)$. The percentage of overweight and obesity started to be significant in the 30-39 year age group. Moreover, the OR for obesity ranged from 2.7 to $7.0(p<0.05-0.01)$ for those women with only elementary education. Besides, high parity was significantly associated with obesity and elementary education. For chronic conditions, the percentages of hypertension, diabetes, hypertriglyceridemia, osteoporosis, and rheumatoid arthritis were significantly correlated with increased BMI in Jordanian women. With age adjustment, however, only hypertension was associated with obese level 3 with OR of 7.2 and $95 \%$ CI of $2.1-25.1(\mathrm{p}<0.01)$.

Conclusion: There is a high prevalence of overweight/obesity among women in Jordan, which was related to high parity and low education level. This high prevalence of obesity increased the incidence of chronic diseases, such as hypertension. Therefore, communitybased multiple strategies are required to combat obesity in Jordanian women.

Keywords: women, obesity, hypertension, chronic diseases, parity, education, Jordan

\section{Introduction}

Obesity and overweight pose a significant public health threat as a rising epidemic in many countries. ${ }^{1}$ In recent years, an increase in body mass index (BMI) was linked to the risk burden of non-communicable chronic diseases globally. ${ }^{2-4}$

Several epidemiologic studies have shown that the rates of obesity in all ages and among both males and females irrespective of geographical locality, ethnicity, or
Correspondence: Mona Bustami

Email mbustami@uop.edu.jo 
socioeconomic status are increasing. ${ }^{5,6}$ For instance, the United States National Health and Nutrition Examination Survey has revealed data showing the overall prevalence of obesity among adults in 2013-2014 was 37.7\%. However, the prevalence of obesity is generally greater in women. ${ }^{7}$ The prevalence among women was $40.4 \%$ compared with $35.0 \%$ among men. ${ }^{8}$ Furthermore, women with high BMI are marked at higher risk of breast cancer, ${ }^{9}$ atherosclerotic cardiovascular disease, ${ }^{10}$ type 2 diabetes, ${ }^{11}$ hypertension, ${ }^{12}$ dyslipidemia, ${ }^{13}$ musculoskeletal disorders, ${ }^{14}$ endocrine disorders, ${ }^{15}$ risk of infertility, ${ }^{16}$ and impaired pulmonary function. ${ }^{17}$

The overcharging aim to prevent overweight and obesity among women has been highly addressed to governments by the world health organization (WHO) to prevent premature death due to chronic diseases. ${ }^{18}$ Aging along with obesity are considered to be the most popular universal contributors to health decline. During 2016, 78\% of the total 36,000 Jordanian deaths occurred because of chronic diseases. ${ }^{19}$ In addition, it was estimated that the prevalence of chronic diseases from 2005 to 2050 in Jordan is expected to accelerate rapidly. ${ }^{20}$

In a 2008 study, the age-standardized prevalence of obesity in the northern area of Jordan was $28.1 \%$ in men and $53.1 \%$ in women. ${ }^{21}$ Besides, the same study reported increased obesity percentages in women aged $\geq 40$ years in 2008 compared to the surveys performed in 1994 and $2004 .^{21}$ As in other areas, obesity in Jordan coincided with an increased odds ratio of diabetes, hypertension, and dyslipidemia. $^{21-23}$ Though, weight reduction and control should be the center of secondary prevention strategies of hypertension and cardiovascular disease when it comes to conveying clinical information into practice.

Recently, we have studied the factors associated with natural menopause age, mainly related to premature and early menopause among Jordanian women. ${ }^{24}$ Although the BMI increase was not a factor in premature or early menopause in the latter study, BMI data in women was alarming. Therefore, in the present study, we investigated the prevalence of obesity in relation to age, education, and parity, and its correlation to chronic diseases and its emerging risk factors in Jordanian women.

\section{Materials and Methods}

\section{Study Approval, Design, and Subjects}

This study was designed for cross-sectional research. A study protocol was submitted to the Research Ethics Committee at the Jordan University Hospital and received approval (Approval \#38/2015) in early 2016. This study was carried out at Jordan University Hospital per the Declaration of Helsinki, whereby every subject has agreed to enroll by willingly signing informed consent.

\section{Subjects and the Selection Process}

For the selection process, we followed a random drop-off technique to the Obstetrics and Gynecology clinics atJordan University Hospital. Any female 18 years of age and older was asked to enroll in the study. Investigators discussed the importance of the study to each participant. Following the acceptance to enroll, each participant signed informed consent. Furthermore, the sample size was calculated to be $\geq 380$ subjects, based on $50 \%$ probability of obesity in Jordan, 0.05 error and $95 \%$ confidence interval $(95 \% \mathrm{CI}) .^{21}$

Four hundred and sixty-eight accepted to enroll in the study. Out of the 468, 40 subjects were excluded from the analysis because they were non-Jordanian and were residing in Jordan as students, ie, they were residing for a short period of time.

With the help of the health-trained investigators, each participant answered a questionnaire containing 30 questions. The questions have information about each participant's socioeconomic factors, including marital status, having kids, type of work, household salary, and education status. Furthermore, the questionnaire included data on each subject's smoking habits, chronic conditions, and diseases.

\section{Body Mass Index (BMI)}

Each subject's weight and height were measured and recorded (Seca 700 with stadiometer, Hamburg, Germany). BMI was calculated and classified (c-BMI) using the International Classification recommended by WHO. c-BMI was as follows: 1 for underweight $\left(<18.5 \mathrm{~kg} / \mathrm{m}^{2}\right), 2$ for standard weight $\left(18.5-24.9 \mathrm{~kg} / \mathrm{m}^{2}\right)$, 3 for overweight $\left(25.0-29.9 \mathrm{~kg} / \mathrm{m}^{2}\right), 4$ for obese 1 (30$\left.34.9 \mathrm{~kg} / \mathrm{m}^{2}\right), 5$ for obese $2\left(35-39.9 \mathrm{~kg} / \mathrm{m}^{2}\right)$, and 6 for obese $3\left(>40 \mathrm{~kg} / \mathrm{m}^{2}\right)$.

\section{Data Analysis}

Means and standard deviations were used to describe the continuous variables and percentages to describe categorical variables. Each categorical variable's frequencies were reported, and the 95\% confidence interval (95\% CI) for each variable was calculated. For association analysis, Chisquare analysis was used with the estimation of odds ratio (OR) and 95\% CI. Furthermore, multinomial logistic regression analysis was applied to a combination of independent 
variables and a dependent condition with covariate factors, such as age and socioeconomic variables. A p-value of $<0.05$ was considered to be statistically significant. All analyses were performed using SPSS 25 statistical package.

\section{Results}

\section{Sociodemographic Characteristics and \\ Health Status of the Study Population}

The study population consisted of 428 Jordanian women with a mean age of 48.6 years $( \pm 14.5 \mathrm{SD})$ (Table 1$)$. The majority were married $(75.5 \%)$, having kids $(68.5 \%)$, housewives $(64 \%)$, never smoked $(79.2 \%)$, and their household income was low $(75.2 \%)$. Besides, half of the population $(50.9 \%)$ had a college degree, and slightly higher than half (57.7\%) considered having good health (Table 1).

More than half $(59.8 \%)$ of the study population had arthritis pain. However, the most common chronic condition that affected the study group was hypertension (29.5\%), and to a lesser extent, diabetes and hypertriglyceridemia (Table 2). Furthermore, women with ovarian

Table I Characteristics of the Study Population

\begin{tabular}{|c|c|c|c|}
\hline Parameter & Description & Number & $\begin{array}{l}\text { Percentage } \\
\text { (\%) }\end{array}$ \\
\hline Age (yr) & $48.6 \pm 14.5$ & 428 & $100 \%$ \\
\hline \multirow[t]{2}{*}{ Marital Status } & Single & 104 & 24.3 \\
\hline & Married/been married & 324 & 75.7 \\
\hline Having Kids & Yes & 293 & 68.5 \\
\hline \multirow[t]{3}{*}{ Education } & Elementary & 150 & 35.1 \\
\hline & High School & 60 & 14.0 \\
\hline & College & 218 & 50.9 \\
\hline \multirow[t]{4}{*}{ Type of Work } & Employee & 94 & 22.0 \\
\hline & Self-Employed & 4 & 1.2 \\
\hline & Housewife & 274 & 64.0 \\
\hline & Student & 55 & 12.9 \\
\hline Household & $<500 \mathrm{JD}$ & 303 & 70.8 \\
\hline \multirow[t]{2}{*}{ Income (JD) } & 500-999 & 89 & 20.8 \\
\hline & $>1000$ & 35 & 8.2 \\
\hline \multirow[t]{4}{*}{ Smoking } & Current & 50 & 11.7 \\
\hline & Sometimes & 10 & 2.3 \\
\hline & Past & 29 & 6.8 \\
\hline & Never & 339 & 79.2 \\
\hline \multirow[t]{4}{*}{ Health } & Excellent & 92 & 21.5 \\
\hline & Good & 155 & 36.2 \\
\hline & Average & 136 & 31.8 \\
\hline & Poor & 42 & 9.8 \\
\hline
\end{tabular}

Table 2 Frequency of Chronic Diseases/Conditions in a Sample of Jordanian Women with an Age Range of 20-75 Years

\begin{tabular}{|l|l|l|l|}
\hline \multirow{2}{*}{ Diseases } & Number & Percentage & \multirow{2}{*}{ Cl 95\% } \\
\cline { 2 - 3 } & & $\mathbf{n}=\mathbf{4 2 8}$ & \\
\hline Arthritis pain & 257 & 59.8 & $57.0-66.0$ \\
Hypertension & 127 & 29.5 & $24.3-34.1$ \\
Diabetes & 77 & 17.9 & $14.4-21.9$ \\
Hypertriglyceridemia & 69 & 16.0 & $12.3-19.9$ \\
Ovarian Cysts & 62 & 14.4 & $11.2-18.1$ \\
Uterine Fibrosis & 62 & 14.4 & $11.2-18.1$ \\
Osteoporosis & 52 & 12.1 & $9.2-15.6$ \\
Rheumatoid arthritis & 21 & 4.9 & $3.0-7.4$ \\
Heart diseases & 18 & 4.2 & $1.5-6.5$ \\
Hysterectomy & 17 & 4.0 & $2.3-6.3$ \\
Hypercholesterolemia & 13 & 3.0 & $1.4-4.2$ \\
Thyroid diseases & 4 & 0.9 & $0.6-1.4$ \\
Hepatitis B virus infection & 3 & 0.7 & $0.4-1.1$ \\
Kidney diseases & 3 & 0.7 & $0.4-1.1$ \\
Migraine & 2 & 0.5 & $0.3-0.8$ \\
Asthma & 2 & 0.5 & $0.3-0.8$ \\
Gout & 2 & 0.5 & $0.3-0.8$ \\
Leukemia & 2 & 0.5 & $0.3-0.8$ \\
Ovariectomy & 2 & 0.5 & $0.3-0.8$ \\
Bone Marrow Fibrosis & 1 & 0.2 & $0.1-0.3$ \\
Breast Fibrosis & 1 & 0.2 & $0.1-0.3$ \\
Epilepsy & 1 & 0.2 & $0.1-0.3$ \\
Hyper colon & 1 & 0.2 & $0.1-0.3$ \\
Mediterranean fever & 1 & 0.2 & $0.1-0.3$ \\
Systemic lupus erythematosus & 1 & 0.2 & $0.1-0.3$ \\
Turner syndrome & $\mathrm{I}$ & 0.2 & $0.1-0.3$ \\
\hline
\end{tabular}

cysts, uterine fibrosis, and osteoporosis were $12-14 \%$. On the other hand, the percentage of autoimmune-related diseases, such as rheumatoid arthritis, systemic lupus erythematosus, and thyroid diseases was $6.1 \%$.

\section{Prevalence of Overweight and Obesity in Jordanian Women and Its Relation to Age, Education Level and Parity}

The percentage frequency of the study population revealed that the majority of women were overweight (Figure 1A). This represented an overall c-BMI average of $3.25( \pm 1.19)$ with a $95 \% \mathrm{CI}$ of $3.14-3.37$. According to c-BMI $\geq 3$, the age-standardized prevalence of overweight/obese Jordanian women was $70.6 \%$ (95\% CI $66.0-74.8 \%)$. On the other hand, the age-standardized prevalence of only obese women was 36.4 (95\% Cl 31.9-41.2\%). Furthermore, data showed that as females aged, the c-BMI significantly increased (Figure 1B). According to the age groups, the percentage of overweight/obese females increased from $\sim 26 \%$ in $20-29$-year age groups to a range between $73-$ 

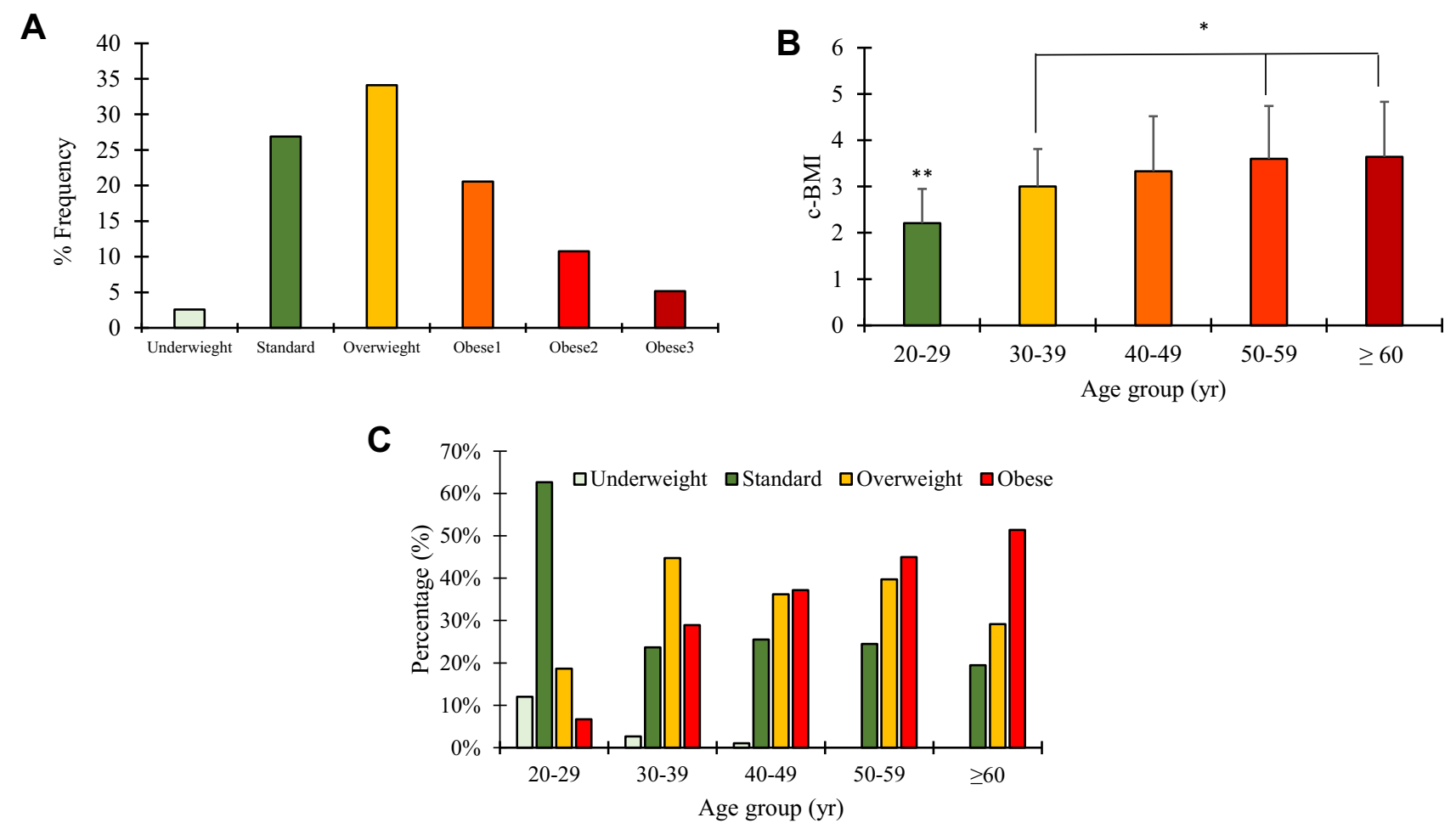

Figure I (A) The distribution frequency of all subjects according to c-BMI. (B) The mean of c-BMI in each age group (error bars represent standard deviation), and **Means significantly different than all other age groups $(p<0.01)$; *Means significantly different than $50-59$ and $\geq 60$ years groups $(p<0.05)$. $(\mathbf{C})$ The distribution percentages of underweight, standard, overweight, and obese women in Jordan according to their age.

$84 \%$ in 30 years of age and older $(\mathrm{p}<0.0001)$ (Figure $1 \mathrm{C})$. However, the percentage of women with the standard weight (c-BMI of 2) was kept constant ( $20 \%$ ) from 30 years of age and above. On the other hand, the percentage of obese women kept increasing with age (Figure 1C).

To evaluate if the education level, having kids, or smoking status were an associated factor with prevalence of obesity in Jordanian women, we used a multinomial regression analysis adjusted with age as a covariate factor. The results showed that obesity, but not overweight, was related to low education levels (Table 3 ). The adjusted OR (aOR) values ranged from 2.7 to 7.0 for elementary education and obesity, and these aOR values became insignificant with high school and college educations. On the other hand, no significant correlation between high BMI and having kids or smoking status was observed.

Furthermore, when parity was classified to low (1-2 kids), medium (3-4 kids), grand parity (5-6 kids) and high grand parity ( $>7$ kids), obesity was significantly associated with medium, grand, and high grand parity in comparison to low parity ( $\mathrm{p}<0.05,0.05,0.01$, respectively) (Table 4). When age was introduced as a cofactor, only high grand parity was associated with obesity (Table 4). However, when education was introduced, no significance was noted. Besides, low education was significantly correlated with an increase in parity (Table 5).

\section{Association Between Overweight/ Obesity and Chronic Conditions}

Since overweight/obesity was a major observational factor in the Jordanian population, further analysis related to chronic/disease conditions with high BMI was performed (Table 6). The percentages of hypertension, diabetes, hypertriglyceridemia, osteoporosis, and rheumatoid arthritis were significantly correlated with increased c-BMI in Jordanian women. Furthermore, the OR ranged between 2.53 and 2.76 for metabolic syndrome-related conditions and was extremely high (9.01) for rheumatoid arthritis.

After adjusting for age and socioeconomic variables in the multinomial regression analysis, only hypertension was associated with an obese 3 level and an aOR of 7.2 (Table 7). Furthermore, rheumatoid arthritis showed a trend towards correlation with overweight and obese 3 (aOR 7.6 and 10.3, $\mathrm{p}=0.058$ and 0.056 , respectively). On the other hand, diabetes, osteoporosis, and hypertriglyceridemia became insignificantly correlated with overweight/obesity (Table 7). 
Table 3 Multinomial Regression Analysis with OR and $\mathrm{Cl}-95 \%$ of BMI Type and Education Level Adjusted with Age

\begin{tabular}{|c|c|c|c|c|}
\hline BMI & Education & aOR* & $95 \% \mathrm{Cl}$ & P value \\
\hline Standard & & Reference & Reference & Reference \\
\hline \multirow[t]{3}{*}{ Overweight } & Elementary & 1.5 & $0.8-3.1$ & 0.238 \\
\hline & High School & 1.3 & $0.6-2.8$ & 0.517 \\
\hline & College & - & - & - \\
\hline \multirow[t]{3}{*}{ Obese I } & Elementary & 3.4 & I.6-7.5 & 0.002 \\
\hline & High School & 1.8 & $0.7-4.3$ & 0.211 \\
\hline & College & - & - & - \\
\hline \multirow[t]{3}{*}{ Obese 2} & Elementary & 2.7 & $1.1-6.7$ & 0.036 \\
\hline & High School & 1.8 & $0.6-5.3$ & 0.323 \\
\hline & College & - & - & - \\
\hline \multirow[t]{3}{*}{ Obese 3} & Elementary & 7.0 & $1.9-26.5$ & 0.004 \\
\hline & High School & 2.8 & $0.6-14.4$ & 0.211 \\
\hline & College & - & - & - \\
\hline
\end{tabular}

Note: *Adjusted OR.

\section{Discussion}

The present study showed that age-adjusted prevalence of overweight and obesity is high (70.6\%) in Jordanian women. This is similar to females living in the eastern Mediterranean regions, including Jordan. ${ }^{25,26}$ However, the age-adjusted prevalence for obesity is moderately high $(36.4 \%)$. The latter is less than the reported study in northern Jordan, ${ }^{21}$ or in Kuwait, Saudi Arabia, or Qatar, but closer to women from Lebanon, Iran, or Tunisia. ${ }^{25}$ Furthermore, our findings stressed on the association of obesity to low education level and high parity. Therefore, a good reason for the differences in the prevalence of obesity in the literature between different populations and studies is the percentage of women with low education levels and the number of parity each woman had in each study. Furthermore, we showed that BMI increased steadily from the age group of 30-39 to over 60 years. Hence, making it evident that increasing BMI is highly associated with age. This finding is expected since previous studies showed that overweight/obesity is related to age. ${ }^{27}$

There were no coinciding studies in Jordan that correlate educational level to obesity among women. However, previous research has shown that the prevalence of overweight/ obesity among low socioeconomic levels. ${ }^{21,26}$ Herein, we showed that the prevalence of obesity was inversely related to educational levels. Women with only elementary education showed to be the highest in obesity. This is in agreement with previous studies that have produced ample evidence that obesity is related to low educational levels, ${ }^{28-30}$ and consequently such low education would disrupt the dynamic factor of the socioeconomic ladder. ${ }^{31}$

Since an increasing number of pregnancies may contribute to postpartum weight retention, ${ }^{32,33}$ this study showed that increasing parity is significantly related to obesity. ${ }^{34,35}$ Furthermore, as a proxy for socioeconomic status, low educational level is inversely associated with parity, and when cofounded with obesity, lower education was highly related to increased odds of obesity due to increase in parity. ${ }^{34,36}$

Table 4 Multinomial Regression Analysis with OR and Cl-95\% of Obesity and Parity without and with Age Adjustment

\begin{tabular}{|l|l|l|l|l|}
\hline Parity & OR & P value & aOR * & P value \\
\hline Grand Parity & $4.26(1.64-11.1)$ & $<0.01$ & $3.13(1.09-9.02)$ & $<0.05$ \\
High Parity & $3.19(1.27-8.00)$ & $<0.05$ & $2.57(0.97-6.8 I)$ & 0.058 \\
Medium Parity & $2.8 I(1.07-7.38)$ & $<0.05$ & $2.50(0.93-6.67)$ & 0.068 \\
Low Parity & Reference & - & Reference & - \\
\hline
\end{tabular}

Note: *Adjusted OR.

Table 5 Number of Women and Their Parity in Comparison to Their Education Level

\begin{tabular}{|l|l|l|l|l|l|l|}
\hline \multirow{2}{*}{ Education } & \multicolumn{5}{|c|}{ Parity } & \multirow{2}{*}{ Total } \\
\cline { 2 - 7 } & High Grand Parity & Grand Parity & Medium Parity & Low Parity & Null Parity \\
\hline Elementary & $64 * * *$ & $40^{* * *}$ & $23 *$ & 7 & 16 & 150 \\
High school & $13 * *$ & $15^{*}$ & $18 *$ & 6 & 8 & 60 \\
College & 11 & 27 & 37 & 37 & 106 & 218 \\
Total & 88 & 82 & 78 & 50 & 130 & 428 \\
\hline
\end{tabular}

Note: ${ }^{*} \mathrm{p}<0.05, * * \mathrm{p}<0.01, * *{ }^{*} \mathrm{p}<0.001$ in comparison to low parity. 
Table 6 Association of Overweight/Obese Jordanian Women with Chronic Conditions/Diseases

\begin{tabular}{|c|c|c|c|c|c|c|}
\hline Diseases & Number & $\begin{array}{l}\text { Number of Cases } \\
\text { with High BMI }\end{array}$ & $\begin{array}{l}\text { Percentage of } \\
\text { Cases to High BMI* }\end{array}$ & OR & Cl $95 \%$ & $\mathbf{P}$ value $* *$ \\
\hline Arthritis pain & 257 & 173 & 57.3 & 0.76 & $0.49-1.16$ & 0.204 \\
\hline Hypertension & 127 & 106 & 35.1 & 2.70 & $1.60-4.57$ & $<0.001$ \\
\hline Diabetes & 77 & 64 & 21.2 & 2.34 & $1.24-4.42$ & $<0.01$ \\
\hline Hypertriglyceridemia & 69 & 58 & 19.2 & 2.49 & $|.28-4.9|$ & $<0.01$ \\
\hline Ovarian Cysts & 62 & 50 & 16.6 & 1.92 & $0.98-3.74$ & 0.06 \\
\hline Uterine Fibrosis & 62 & 50 & 16.6 & 1.89 & $0.97-3.68$ & 0.06 \\
\hline Osteoporosis & 52 & 43 & 14.2 & 2.19 & $1.02-4.57$ & $<0.05$ \\
\hline Rheumatoid arthritis & 21 & 20 & 6.6 & 8.87 & $1.18-66.79$ & $<0.01$ \\
\hline Hysterectomy & 17 & 15 & 5.0 & 3.24 & $0.73-14.38$ & 0.171 \\
\hline Heart diseases & 18 & 15 & 5.0 & 2.14 & $0.6 \mathrm{I}-7.54$ & 0.224 \\
\hline Hypercholesterolemia & 13 & 8 & 2.6 & 0.66 & $0.21-2.05$ & 0.469 \\
\hline
\end{tabular}

Notes: *Number of women with high BMI is 302. **Chi square test (2-sided).

Table 7 Multinomial Regression Analysis with OR and Cl-95\% of BMI Type to Chronic Conditions Adjusted with Age and Socioeconomic Variables*

\begin{tabular}{|c|c|c|c|c|c|}
\hline BMI & Hypertension & Diabetes & Hypertriglyceridemia & Rheumatoid Arthritis & Osteoporosis \\
\hline Standard $18-24.9 \mathrm{~kg} / \mathrm{m}^{2}$ & Reference & Reference & Reference & Reference & Reference \\
\hline \multirow[t]{2}{*}{ Overweight $25-29.9 \mathrm{~kg} / \mathrm{m}^{2}$} & $0.6(0.3-1.2)$ & $0.8(0.4-1.8)$ & $1.3(0.6-2.9)$ & $7.6(0.9-61.4)$ & $1.2(0.9-2.9)$ \\
\hline & & & & $\mathrm{p}=0.058$ & \\
\hline Obese I $30-34.9 \mathrm{~kg} / \mathrm{m}^{2}$ & I.I (0.5-2.4) & $1.2(0.5-2.7)$ & $1.0(0.4-2.4)$ & $3.4(0.4-33.3)$ & I.I (0.4-2.9) \\
\hline \multirow[t]{2}{*}{ Obese $235-39.9 \mathrm{~kg} / \mathrm{m}^{2}$} & $2.2(0.9-5.3)$ & $1.5(0.6-3.7)$ & $1.2(0.4-3.2)$ & $2.6(0.2-31.7)$ & $0.8(0.3-2.5)$ \\
\hline & $\mathrm{P}=0.083$ & & & & \\
\hline \multirow[t]{2}{*}{ Obese $3 \geq 40 \mathrm{~kg} / \mathrm{m}^{2}$} & $7.2(2.1-25 . I)$ & $1.3(0.4-4.2)$ & $2.0(0.6-6.5)$ & $10.3(0.9-113.0)$ & $1.2(0.3-4.5)$ \\
\hline & $P<0.01$ & & & $p=0.056$ & \\
\hline
\end{tabular}

Note: *Marital status, having kids, type of work, and education.

Aging, with its related consequences on health, is considered a significant contributor to health decline. When the accumulation of unhealthy lifestyle behaviors such as low physical activity, high intake of sugar-sweetened beverages, and low intake of fruits and vegetables during aging would increase multiple inflammatory processes and therefore, linkage to chronic inflammatory diseases occurs. ${ }^{37-40}$ In the present study, hypertension, diabetes, hypertriglyceridemia, osteoporosis, and rheumatoid arthritis were all significantly correlated with increased BMI and age. However, after adjusting for age and socioeconomic variables, only hypertension was associated with obesity. The latter points to other risk factors accumulated with aging that may enhance the prevalence of diabetes, hypertriglyceridemia, osteoporosis, and rheumatoid arthritis.
Furthermore, this reveals the limitations of the present study, which did not include variables such as lifestyle behavior for each subject, obesity family history, and obesity in early adolescence. ${ }^{41}$ Besides, this study is a cross-sectional observational study, and it is difficult to make inferences regarding causal relationships and obesity. Furthermore, although the sample size of this study is adequate, the number of subjects enrolled is limited. Finally, some of the questionnaire questions were relatively subjective and depended on each participant's opinion.

Obesity is an alarming risk factor for hypertension, leading to increased morbidity and mortality among Jordanian women. Hypertension is a modifiable risk factor for cardiovascular and renal diseases. ${ }^{42}$ Besides, multiple pathophysiological abnormalities were found to be 
highly related to hypertension and obesity. The associated abnormalities are inflammation, insulin resistance, increased oxidative stress, abnormal adipokines production, the sympathetic nervous system, and the reninangiotensin-aldosterone systems. ${ }^{43}$ Thus, early diagnosis and treatment of obesity impact the prevention of hypertension and its consequences. Furthermore, obesity is preventable by improving our understanding of the factors that promote obesity from an early age. Thus, implementing preventing obesity into clinical practice should increase awareness against high-fat food consumption, reduced physical exercise, and improved sedentary lifestyle. ${ }^{44,45}$ Medical education in the clinic regarding obesity and its correlation to metabolic and cardiovascular diseases, adopting new lifestyle habits for weight management should be of high importance. Furthermore, this and other previous studies should help health policymakers implement awareness of the background of obesity in Jordan.

In conclusion, there is a high prevalence of overweight/ obesity among women in Jordan, which was related to high parity and low education level. Besides, this high prevalence increased the incidence of chronic conditions among obese women. Therefore, community-based multiple strategies are required to combat obesity in Jordanian women. Furthermore, research should be dedicated to supporting women's well-being in Jordan's clinics by elevating the association of overweight and obesity with increased morbidity and mortality rates. Finally, it is vital to improve our understanding of obesity in women to initiate interventional strategies to control chronic diseases.

\section{Abbreviations}

95\% CI, 95\% confidence interval; aOR, adjusted odds ratio; BMI, body mass index; c-BMI, classified BMI; OR, odds ratio; WHO, World Health Organization.

\section{Acknowledgments}

The authors acknowledge the financial support from the Deanship of Research and Graduate Studies at the University of Petra (Grant Numbers 5-4-2015), Amman, Jordan, and Jordan University Hospital to facilitate meeting the participants and collecting samples. A special gratitude acknowledgment dedicated to Ms. Suzan Alwawi, University of Petra, for technical assistance and finally to all study participants. Khalid Z Matalka reports their current address is Matalka's Scientific Writing, Lexington, MA, 02420, USA.

\section{Author Contributions}

All authors made substantial contributions to conception and design, acquisition of data, or analysis and interpretation of data; took part in drafting the article or revising it critically for important intellectual content; agreed to submit to the current journal; gave final approval for the version to be published; and agreed to be accountable for all aspects of the work.

\section{Disclosure}

The authors declare no conflicts of interest for this work.

\section{References}

1. Afshin A, Forouzanfar MH, et a; GBD 2015 Obesity Collaborators. Health effects of overweight and obesity in 195 countries over 25 years. $N$ Engl J Med. 2017;377(1):13-27. doi:10.1056/NEJMoa1 614362

2. World Health Organization. Obesity and Overweight. Geneva, Switzerland: WHO; 2020. Available from https://www.who.int/news room/fact-sheets/detail/obesity-and-overweight. Accessed March, 20, 2020.

3. Jones A, Bentham G, Foster C, Hillsdon M, Panter J. Tackling Obesities: Future Choices-Obesogenic Environments-Evidence Review. London: Government Office for Science; 2007.

4. Williams EP, Mesidor M, Winters K, Dubbert PM, Wyatt SB. Overweight and obesity: prevalence, consequences, and causes of a growing public health problem. Curr Obes Rep. 2015;4 (3):363-370. doi:10.1007/s13679-015-0169-4

5. Arroyo-Johnson C, Mincey KD. Obesity epidemiology worldwide. Gastroenterol Clin North Am. 2016;45(4):571-579. doi:10.1016/j. gtc.2016.07.012

6. Jastreboff AM, Kotz CM, Kahan S, Kelly AS, Heymsfield SB. Obesity as a disease: the obesity society 2018 position statement. Obesity. 2019;27(1):7-9. doi:10.1002/oby.22378

7. Chooi YC, Ding C, Magkos F. The epidemiology of obesity. Metabolism. 2019;92:6-10. doi:10.1016/j.metabol.2018.09.005

8. Flegal KM, Kruszon-Moran D, Carroll MD, Fryar CD, Ogden CL. Trends in obesity among adults in the United States, 2005 to 2014. JAMA. 2016;315(21):2284-2291. doi:10.1001/jama.2016.6458

9. Picon-Ruiz M, Morata-Tarifa C, Valle-Goffin JJ, Friedman ER, Slingerland JM. Obesity and adverse breast cancer risk and outcome: mechanistic insights and strategies for intervention. CA Cancer J Clin. 2017;67(5):378-397. doi:10.3322/caac.21405

10. Garcia M, Mulvagh SL, Merz CN, Buring JE, Manson JE. Cardiovascular disease in women: clinical perspectives. Circ Res. 2016;118(8):1273-1293. doi:10.1161/CIRCRESAHA.116.307547

11. Maskarinec G, Jacobs S, Park SY, et al. Type II diabetes, obesity, and breast cancer risk: the multiethnic cohort. Cancer Epidemiol Biomarkers Prev. 2017;26(6):854-861. doi:10.1158/1055-9965.EPI16-0789

12. Seravalle G, Grassi G. Obesity and hypertension. Pharmacol Res. 2017;122:1-7. doi:10.1016/j.phrs.2017.05.013

13. Palmer MK, Toth PP. Trends in lipids, obesity, metabolic syndrome, and diabetes mellitus in the United States: an NHANES analysis (2003-2004 to 2013-2014). Obesity. 2019;27(2):309-314. doi:10.1002/oby. 22370 
14. Anandacoomarasamy A, Caterson I, Sambrook P, Fransen M, March L. The impact of obesity on the musculoskeletal system. Int J Obes. 2008;32(2):211-222. doi:10.1038/sj.ijo.0803715

15. Joham AE, Palomba S, Hart R. Polycystic ovary syndrome, obesity, and pregnancy. Semin Reprod Med. 2016;34(2):93-101. doi:10.1055/ s-0035-1571195

16. Silvestris E, de Pergola G, Rosania R, Loverro G. Obesity as disruptor of the female fertility. Reprod Biol Endocrinol. 2018;16(1):22. doi:10.1186/s12958-018-0336-z

17. Dietz W, Santos-Burgoa C. Obesity and its implications for COVID-19 mortality. Obesity. 2020;28(6):1005. doi:10.1002/ oby. 22818

18. World Health Organization. Global action plan for the prevention and control of NCDs 2013-2020. 2013 [cited February 12, 2020]. Available from: https://www.who.int/nmh/publications/ncd-actionplan/en/. Accessed June 16, 2021.

19. World Health Organization. Noncommunicable diseases country profiles 2018. 2018. Available from: https://www.who.int/nmh/publica tions/ncd-profiles-2018/en/. Accessed June 16, 2021.

20. Population Division of the Department of Economic and Social Affairs of the United Nations Secretariat. World Population Prospects: The 2006 Revision. New York (NY): United Nations; 2007.

21. Khader Y, Batieha A, Ajlouni H, El-Khateeb M, Ajlouni K. Obesity in Jordan: prevalence, associated factors, comorbidities, and change in prevalence over ten years. Metab Syndr Relat Disord. 2008;6 (2):113-120. doi:10.1089/met.2007.0030

22. Abujbara M, Batieha A, Khader Y, Jaddou H, El-Khateeb M, Ajlouni K. The prevalence of dyslipidemia among Jordanians. J Lipids. 2018;2018:6298739. doi:10.1155/2018/6298739

23. Khader Y, Batieha A, Jaddou H, et al. Hypertension in Jordan: prevalence, awareness, control, and its associated factors. Int J Hypertens. 2019;2019:3210617. doi:10.1155/2019/3210617

24. Bustami M, Matalka KZ, Elyyan Y, et al. Age of natural menopause among Jordanian women and factors related to premature and early menopause. Risk Manag Healthc Policy. 2021;14:199-207. doi:10.2147/RMHP.S289851

25. Ataey A, Jafarvand E, Adham D, Moradi-Asl E. The relationship between obesity, overweight, and the human development index in World Health Organization Eastern Mediterranean region countries. J Prev Med Public Health. 2020;53(2):98-105. doi:10.3961/ jpmph.19.100

26. Ajlouni K, Khader Y, Batieha A, Jaddou H, El-Khateeb M. An alarmingly high and increasing prevalence of obesity in Jordan. Epidemiol Health. 2020;42:e2020040. doi:10.4178/epih.e2020040

27. Jura M, Kozak LP. Obesity and related consequences to ageing. Age. 2016;38(1):23. doi:10.1007/s11357-016-9884-3

28. Ogden CL, Fakhouri TH, Carroll MD, et al. Prevalence of obesity among adults, by household income and education - United States, 2011-2014. MMWR Morb Mortal Wkly Rep. 2017;66 (50):1369-1373. doi:10.15585/mmwr.mm6650a1

29. Jimenez-Mora MA, Nieves-Barreto LD, Montaño-Rodríguez A, Betancourt-Villamizar EC, Mendivil CO. Association of overweight, obesity and abdominal obesity with socioeconomic status and educational level in Colombia. Diabetes Metab Syndr Obes. 2020;13:1887-1898. doi:10.2147/DMSO.S244761

30. Fouad M, Rastam S, Ward K, Maziak W. Prevalence of obesity and its associated factors in Aleppo, Syria. Prev Control. 2006;2 (2):85-94. doi:10.1016/j.precon.2006.09.001
31. Mcmillan J, Jones F, Beavis A. A new scale for measuring socioeconomic status in educational research: development and validation of the Australian Socioeconomic Index 2006 (AUSEI06). 2009.

32. Deputy NP, Sharma AJ, Kim SY, Hinkle SN. Prevalence and characteristics associated with gestational weight gain adequacy. Obstet Gynecol. 2015;125(4):773-781. doi:10.1097/AOG.0000000000000739

33. Parker HW, Tovar A, McCurdy K, Vadiveloo M. Associations between pre-pregnancy BMI, gestational weight gain, and prenatal diet quality in a national sample. PLoS One. 2019;14(10):e0224034. doi:10.1371/journal.pone.0224034

34. Koch E, Bogado M, Araya F, et al. Impact of parity on anthropometric measures of obesity controlling by multiple confounders: a cross-sectional study in Chilean women. J Epidemiol Community Health. 2008;62(5):461-470. doi:10.1136/jech.2007.062240

35. Huayanay-Espinoza CA, Quispe R, Poterico JA, Carrillo-Larco RM, Bazo-Alvarez JC, Miranda JJ. Parity and overweight/obesity in Peruvian women. Prev Chronic Dis. 2017;14:E102. doi:10.5888/ pcd14.160282

36. Taghdir M, Alimohamadi Y, Sepandi M, Rezaianzadeh A, Abbaszadeh S, Mahmud FM. Association between parity and obesity: a cross sectional study on 6447 Iranian females. J Prev Med Hyg. 2020;61(3):E476-E481. doi:10.15167/2421-4248/jpmh2020.61.3.1430

37. Berentzen NE, Smit HA, van Rossem L, et al. Screen time, adiposity and cardiometabolic markers: mediation by physical activity, not snacking, among 11-year-old children. Int $J$ Obes. 2014;38 (10):1317-1323. doi:10.1038/ijo.2014.110

38. Malik VS, Popkin BM, Bray GA, Després JP, Willett WC, Hu FB. Sugar-sweetened beverages and risk of metabolic syndrome and type 2 diabetes: a meta-analysis. Diabetes Care. 2010;33(11):2477-2483. doi:10.2337/dc10-1079

39. Furman D, Campisi J, Verdin E, et al. Chronic inflammation in the etiology of disease across the life span. Nat Med. 2019;25 (12):1822-1832. doi:10.1038/s41591-019-0675-0

40. Ng R, Sutradhar R, Yao Z, Wodchis WP, Rosella LC. Smoking, drinking, diet and physical activity-modifiable lifestyle risk factors and their associations with age to first chronic disease. Int J Epidemiol. 2020;49(1):113-130. doi:10.1093/ije/dyz078

41. Tauqeer Z, Gomez G, Stanford FC. Obesity in women: insights for the clinician. J Womens Health. 2018;27(4):444-457. doi:10.1089/ jwh.2016.6196

42. He J, Whelton PK. Elevated systolic blood pressure and risk of cardiovascular and renal disease: overview of evidence from observational epidemiologic studies and randomized controlled trials. Am Heart J. 1999;138(3 Pt 2):211-219. doi:10.1016/s0002-8703(99)70312-1

43. Cohen JB. Hypertension in obesity and the impact of weight loss. Curr Cardiol Rep. 2017;19(10):98. doi:10.1007/s11886-017-0912-4

44. Al-Nsour M, Zindah M, Belbeisi A, Hadaddin R, Brown DW, Walke H. Prevalence of selected chronic, noncommunicable disease risk factors in Jordan: results of the 2007 Jordan behavioral risk factor surveillance survey. Prev Chronic Dis. 2012;9:E25.

45. Centers for Disease Control and Prevention (CDC). Assessing risk factors for chronic disease-Jordan, 2004. Morb Mortal Wkly Rep. 2006;55:653-655. 


\section{Publish your work in this journal}

The Journal of Multidisciplinary Healthcare is an international, peerreviewed open-access journal that aims to represent and publish research in healthcare areas delivered by practitioners of different disciplines. This includes studies and reviews conducted by multidisciplinary teams as well as research which evaluates the results or conduct of such teams or healthcare processes in general. The journal covers a very wide range of areas and welcomes submissions from practitioners at all levels, from all over the world. The manuscript management system is completely online and includes a very quick and fair peer-review system. Visit http://www.dovepress.com/testimonials. php to read real quotes from published authors. 